\title{
REDUCED THEORIES IN NONLINEAR ELASTICITY
}

\author{
MARTA LEWICKA*
}

\begin{abstract}
The purpose of this note is to report on the recent development concerning the analysis and the rigorous derivation of thin film models for structures with nontrivial geometry. This includes: (i) shells with mid-surface of arbitrary curvature, and (ii) plates exhibiting residual stress at free equilibria. In the former setting, we derive a full range of models, some of them previously absent from the physics and engineering literature. The latter phenomenon has been observed in different contexts: growing leaves, torn plastic sheets and specifically engineered polymer gels. After reviewing available results, we list open problems with a promising angle of approach.
\end{abstract}

Key words. non-Euclidean plates, nonlinear elasticity, Gamma convergence, calculus of variations

AMS(MOS) subject classifications. 74K20, 74B20

Elastic materials exhibit qualitatively different responses to different kinematic boundary conditions or body forces. As a first step towards understanding the related evolutionary problem, one studies the minimizers of an appropriate nonlinear elastic energy functional. In sections 1-9 of this short review paper we focus on the recent development regarding the study of wavy patterns in experimentally attained configurations where the tissue endeavors to reach a non-attainable equilibrium through assuming a nonzero stress rest state. In sections $10-13$ we present the available rigorous results for dimension reduction in theories of elastic shells of non-trivial mid-surface geometry. Section 14 contains some open problems.

1. Elastic energy of a growing tissue. Consider a sequence of thin 3d films $\Omega^{h}=\Omega \times(-h / 2, h / 2)$, with an open, bounded and simply connected mid-plate $\Omega \subset \mathbb{R}^{2}$. Each $\Omega^{h}$ undergoes a growth process, described instantaneously by a given smooth tensor $a^{h}=\left[a_{i j}^{h}\right]: \bar{\Omega} \rightarrow \mathbb{R}^{3 \times 3}$ with the property: $\operatorname{det} a^{h}(x)>0$. According to the formalism in [19], the following multiplicative decomposition is postulated:

$$
\nabla u=F a^{h}
$$

for the gradient of a deformation $u: \Omega^{h} \rightarrow \mathbb{R}^{3}$. The tensor $F=\nabla u\left(a^{h}\right)^{-1}$ corresponds to the elastic part of $\nabla u$, and accounts for the reorganization of $\Omega^{h}$ in response to the growth tensor $a^{h}$. Hence, the elastic energy of $u$ depends only on $F$ :

$$
I_{W}^{h}(u)=\frac{1}{h} \int_{\Omega^{h}} W\left(\nabla u\left(a^{h}\right)^{-1}\right) \mathrm{d} x, \quad \forall u \in W^{1,2}\left(\Omega^{h}, \mathbb{R}^{3}\right) .
$$

*University of Minnesota, Department of Mathematics, 206 Church St. S.E., Minneapolis, MN 55455, e-mail: lewicka@math.umn.edu, partially supported by the NSF grants DMS-0707275 and DMS-0846996. 
The energy density $W: \mathbb{R}^{3 \times 3} \longrightarrow \mathbb{R}_{+}$is assumed to be $\mathcal{C}^{2}$ in a neighborhood of $S O(3)$, and to satisfy the following conditions of normalization, frame indifference and nondegeneracy:

$$
\begin{array}{ll}
\exists c>0 \quad \forall F \in \mathbb{R}^{3 \times 3} \quad \forall R \in S O(3) \quad W(R)=0, \quad W(R F)=W(F) \\
& W(F) \geq c \operatorname{dist}^{2}(F, S O(3)) .
\end{array}
$$

We remark that the validity of decomposition (1.1) into an elastic and inelastic part requires that it is possible to separate out a reference configuration. Thus this formalism is relevant for the description of processes such as plasticity, swelling and shrinkage in thin films, or plant morphogenesis. Although our results are valid for thin laminae that might be residually strained by a variety of means, we only consider the one-way coupling of growth to shape and ignore the feedback from shape back to growth. However, it would be very interesting to include this coupling in the model as well, once the basic coupling mechanisms are understood.

2. Non-Euclidean elasticity. We now compare the 'growth tensor' approach with the 'target metric' formalism $[3,16]$. On each $\Omega^{h}$ we assume to be given a smooth Riemannian metric $g^{h}=\left[g_{i j}^{h}\right]$. A deformation $u$ of $\Omega^{h}$ is said to be an orientation preserving realization of $g^{h}$, when $(\nabla u)^{T} \nabla u=$ $g^{h}$ and $\operatorname{det} \nabla u>0$, or equivalently when:

$$
\nabla u(x) \in \mathcal{F}^{h}(x)=\left\{R \sqrt{g^{h}}(x) ; R \in S O(3)\right\} \quad \text { a.e. in } \Omega^{h} .
$$

It is hence instructive to study the energy bounding from below $I_{W}^{h}(u)$ :

$$
\tilde{I}_{\text {dist }}^{h}(u)=\frac{1}{h} \int_{\Omega^{h}} \operatorname{dist}^{2}\left(\nabla u(x), \mathcal{F}^{h}(x)\right) \mathrm{d} x \quad \forall u \in W^{1,2}\left(\Omega^{h}, \mathbb{R}^{3}\right) .
$$

This functional measures the average pointwise deviation of $u$ from orientation preserving realizations of $g^{h}$.

We also consider a more general functional $\tilde{I}_{W}^{h}=\int W(x, \nabla u(x))$ with the inhomogeneous $W$ satisfying frame invariance, normalisation and quadratic growth from below, as in (1.3) but with respect to the energy well $\mathcal{F}^{h}$ given in (2.1).

3. Residual stress. Note that one could define the energy as the difference between the pull-back metric of a deformation $u$ and the given metric: $I_{s t r}^{h}(u)=\int\left|(\nabla u)^{T} \nabla u-g^{h}\right|^{2} \mathrm{~d} x$. However, such 'stretching' functional is not appropriate from the variational point of view, because there always exists $u \in W^{1, \infty}$ such that $I_{\text {str }}^{h}(u)=0$. Further, if the Riemann curvature tensor $R^{h}$ associated to $g^{h}$ does not vanish identically, say $R_{i j k l}^{h}(x) \neq 0$, then $u$ has a 'folding structure' [7]; it cannot be orientation preserving (or reversing) in any open neighborhood of $x$.

As proved in [16], the functionals $I_{W}^{h}, \tilde{I}_{W}^{h}$ and $\tilde{I}_{d i s t}^{h}$ have strictly positive infima for non-flat $g^{h}$, which points to the existence of non-zero stress 
at free equilibria (in the absence of external forces or boundary conditions):

$$
R^{h} \not \equiv 0 \Leftrightarrow \quad \inf \left\{\tilde{I}_{\text {dist }}^{h}(u) ; u \in W^{1,2}\left(\Omega^{h}, \mathbb{R}^{3}\right)\right\}>0 .
$$

It is conjectured that the same principle plays a role in the developmental processes of naturally growing tissues, where the process of growth provides a mechanism for the spontaneous formation of non-Euclidean metrics and consequently leads to complicated morphogenesis of the thin film exhibiting waves, ruffles and non-zero residual stress.

4. Relation to isometric immersions. Several questions arise in the study of the proposed energy functionals. A first one is to determine the scaling of the infimum energy in terms of the vanishing thickness $h \rightarrow 0$.

In [16] we considered the case where $g^{h}$ is given by a tangential Riemannian metric $\left[g_{\alpha \beta}\right]$ on $\Omega$, and is independent of $h$ :

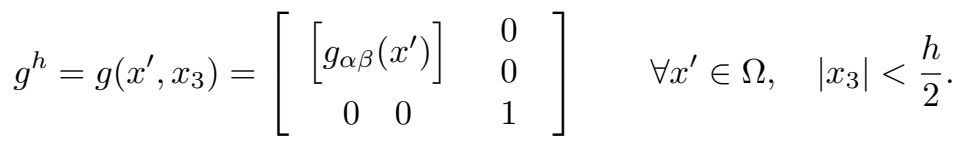

Consequently, if $\left[g_{\alpha \beta}\right]$ has non-zero Gaussian curvature $\kappa_{\left[g_{\alpha \beta}\right]}$, then each $R^{h} \not \equiv 0$, and hence inf $\tilde{I}_{\text {dist }}^{h}(u)>0$ for every $h$. We proved that:

$$
\left[g_{\alpha \beta}\right] \text { has an isometric immersion } y \in W^{2,2}\left(\Omega, \mathbb{R}^{3}\right) \Leftrightarrow \inf \tilde{I}_{\text {dist }}^{h} \leq C h^{2},
$$

for a uniform constant $C$. It also follows that $\kappa_{\left[g_{\alpha \beta}\right]} \not \equiv 0$ if and only if $h^{-2} \inf \tilde{I}_{\text {dist }}^{h} \geq c>0$. Existence of isometric immersions is a longstanding problem in differential geometry, depending heavily on the regularity [8]. Here, we deal with $W^{2,2}$ immersions not studied previously.

5. The prestrained Kirchhoff model. Let us remark that the particular choice of the metric (4.1) is motivated by the experiment reported in [10]. The experiment consisted in fabricating programmed flat disks of gels having a non-constant monomer concentration which induces a 'differential shrinking factor'. The disk was then activated in a temperature raised above a critical threshold, whereas the gel shrunk with a factor proportional to its concentration. This process defined a new target metric on the disk, of the described form and radially symmetric. The metric forced a $3 \mathrm{~d}$ configuration in the initially planar plate, and one of the most remarkable features of this deformation is the onset of some transversal oscillations, which broke the radial symmetry.

Another interesting question in the setting, solved in [16], is to find the limiting zero-thickness theory under the obtained scaling law. Namely, we proved that any sequence of deformations $u^{h}$ with $\tilde{I}_{W}^{h}\left(u^{h}\right) \leq C h^{2}$, converges to a $W^{2,2}$ regular isometric immersion $y$ of $\left[g_{\alpha \beta}\right]$. Conversely, every $y$ with this property can be recovered as a limit of $u^{h}$ whose energy 
scales like $h^{2}$. The $\Gamma$-limit of the rescaled energies is a curvature functional on the space of all $W^{2,2}$ realizations $y$ of $\left[g_{\alpha \beta}\right]$ in $\mathbb{R}^{3}$ :

$$
\frac{1}{h^{2}} \tilde{I}_{W}^{h} \stackrel{\Gamma}{\longrightarrow} \frac{1}{24} \int_{\Omega} \mathcal{Q}_{2}\left(x^{\prime}\right)\left({\sqrt{\left[g_{\alpha \beta}\right]}}^{-1}(\nabla y)^{T} \nabla \vec{n}\right) \mathrm{d} x^{\prime},
$$

Here $\vec{n}$ is the unit normal to the surface $y(\Omega)$, while $\mathcal{Q}_{2}\left(x^{\prime}\right)$ are the quadratic forms, nondegenerate and positive definite on the symmetric $2 \times 2$ tensors, which can be calculated explicitly (see [16]) from $\nabla^{2} W\left(x^{\prime}, \sqrt{\left[g_{\alpha \beta}\right]}\right)$.

6. Rigidity estimate. As a ingredient of proofs, we give a generalization of the nonlinear rigidity estimate [5] to the non-Euclidean setting. Namely, for all $u \in W^{1,2}\left(\mathcal{U}, \mathbb{R}^{n}\right)$ there exists $Q \in \mathbb{R}^{n \times n}$ such that:

$\int_{\mathcal{U}}|\nabla u(x)-Q|^{2} \mathrm{~d} x \leq C\left(\int_{\mathcal{U}} \operatorname{dist}^{2}(\nabla u, \mathcal{F}(x)) \mathrm{d} x+\|\nabla g\|_{L^{\infty}}^{2}(\operatorname{diam} \mathcal{U})^{2}|\mathcal{U}|\right)$

where $\mathcal{F}=S O(n) \sqrt{g}$ is the energy well relative to a given metric $g$ on $\mathcal{U}$. The constant $C$ depends on $\|g\|_{L^{\infty}},\left\|g^{-1}\right\|_{L^{\infty}}$, and on $\mathcal{U}$, uniformly for a family of domains which are uniformly bilipschitz equivalent.

Note that in case $g=\mathrm{Id}$, the infimum of $I_{\text {dist }}^{h}$ in (2.2) is naturally 0 and is attained only by the rigid motions. In our setting, since there is no realization of $I_{\text {dist }}^{h}(u)=0$ if the Riemann curvature of $g$ is non-zero, we can only estimate the deviation of the deformation from a linear map at the expense of an extra term, proportional to the gradient of the metric.

7. A hierarchy of scalings. Given a sequence of growth tensors $a^{h}$ defined on $\Omega^{h}$, the general objective is now to analyse the behavior of the minimizers of the corresponding energies $I_{W}^{h}$ as $h \rightarrow 0$.

As a first step in this direction we established in [12] a lower bound on $m_{h}=\inf I_{W}^{h}$ in terms of a power law: $m_{h} \geq c h^{\beta}$, for all values of $\beta$ greater than a critical $\beta_{0}$ in (7.1). This critical exponent depends on the asymptotic behavior of the perturbation $a^{h}-\mathrm{Id}$ in terms of the thickness $h$. Under existence conditions for certain classes of isometries, it can be shown that actually $m_{h} \sim h^{\beta_{0}}$.

Namely, define: $\operatorname{Var}\left(a^{h}\right)=\left\|\nabla_{\tan }\left(a_{\mid \Omega}^{h}\right)\right\|_{L^{\infty}(\Omega)}+\left\|\partial_{3} a^{h}\right\|_{L^{\infty}\left(\Omega^{h}\right)}$, together with the scaling $\omega_{1}=\sup \left\{\omega ; \lim _{h \rightarrow 0} h^{-\omega} \operatorname{Var}\left(a^{h}\right)=0\right\}$ of the variations $\operatorname{Var}\left(a^{h}\right)$ in $h$. Assume that: $\left\|a^{h}\right\|_{L^{\infty}\left(\Omega^{h}\right)}+\left\|\left(a^{h}\right)^{-1}\right\|_{L^{\infty}\left(\Omega^{h}\right)} \leq C$ and $\omega_{1}>0$. Further, assume that for some $\omega_{0} \geq 0$, there exists the limit:

$$
\epsilon_{g}\left(x^{\prime}\right)=\lim _{h \rightarrow 0} \frac{1}{h^{\omega_{0}}} f_{-h / 2}^{h / 2} a^{h}\left(x^{\prime}, t\right)-\operatorname{Id} \mathrm{d} t \quad \text { in } L^{2}\left(\Omega, \mathbb{R}^{3 \times 3}\right) .
$$

which satisfies: $\operatorname{curl}^{T} \operatorname{curl}\left(\epsilon_{g}\right)_{2 \times 2} \not \equiv 0$, and that $\omega_{0}<\min \left\{2 \omega_{1}, \omega_{1}+1\right\}$. Then, for every $\beta$ with:

$$
\beta>\beta_{0}=\max \left\{\omega_{0}+2,2 \omega_{0}\right\},
$$


there holds: $\limsup _{h \rightarrow 0} \frac{1}{h^{\beta}} \inf I_{\text {dist }}^{h}=+\infty$.

We expect it should be possible to rigorously derive a hierarchy of prestrained limiting theories, differentiated by the embeddability properties of the target metrics, encoded in the scalings of (the components of) their Riemann curvature tensors. This is in the same spirit as the different scalings of external forces lead to a hierarchy of nonlinear elastic plate theories displayed in [6]. For shells, that are thin films with mid-surface or arbitrary (non-flat) geometry, an infinite hierarchy of models was proposed, by means of asymptotic expansion in [17], and it remains in agreement with all the rigorously obtained results $[4,13,14,15]$.

8. The prestrained von Kármán model. Towards studying the dynamical growth problem, in [12] we considered the tensors of the form:

$$
a^{h}\left(x^{\prime}, x_{3}\right)=\operatorname{Id}+h^{2} \epsilon_{g}\left(x^{\prime}\right)+h x_{3} \gamma_{g}\left(x^{\prime}\right),
$$

with given matrix fields $\epsilon_{g}, \gamma_{g}: \bar{\Omega} \rightarrow \mathbb{R}^{3 \times 3}$. Note that the assumptions of the scaling result in the previous section do not hold, since in the present case: $\omega_{0}=2 \omega_{1}=\omega_{1}+1=2$.

We proved that, in this setting, $\inf I_{W}^{h} \leq C h^{4}$, while the lower bound $h^{-4} \inf I_{W}^{h} \geq c>0$ is equivalent to:

$$
\operatorname{curl}\left(\left(\gamma_{g}\right)_{2 \times 2}\right) \not \equiv 0 \quad \text { or } \quad 2 \operatorname{curl}^{T} \operatorname{curl}\left(\epsilon_{g}\right)_{2 \times 2}+\operatorname{det}\left(\gamma_{g}\right)_{2 \times 2} \not \equiv 0,
$$

We use the following notational convention: for a matrix $F$, its $n \times m$ principle minor is denoted by $F_{n \times m}$ and the superscript ${ }^{T}$ refers to the transpose of a matrix or an operator.

The two expressions in (8.2) are the (negated) linearized Gauss-Codazzi equations corresponding to the metric $I=\mathrm{Id}+h^{2}\left(\epsilon_{g}\right)_{2 \times 2}$ and the second fundamental form $I I=\frac{1}{2} h\left(\gamma_{g}\right)_{2 \times 2}$ on $\Omega$. Equivalently, the above conditions guarantee that the highest order terms in the expansion of the Riemann curvature tensor components $R_{1213}, R_{2321}$ and $R_{1212}$ of $g^{h}=\left(a^{h}\right)^{T} a^{h}$ do not vanish. Also, either of the conditions implies that inf $\mathcal{I}_{4}>0$ (see definition below), which yields the lower bound on $\inf I_{W}^{h}$.

The $\Gamma$-limit of the rescaled energies is a function of the out-of-plane displacement $v \in W^{2,2}(\Omega, \mathbb{R})$ and in-plane displacement $w \in W^{1,2}\left(\Omega, \mathbb{R}^{2}\right)$ :

$$
\begin{aligned}
& \frac{1}{h^{4}} I_{W}^{h} \stackrel{\Gamma}{\longrightarrow} \mathcal{I}_{4} \quad \text { where } \\
& \begin{aligned}
\mathcal{I}_{4}(w, v)=\frac{1}{24} \int_{\Omega} & \mathcal{Q}_{2}\left(\nabla^{2} v+\frac{1}{2}\left(\gamma_{g}\right)_{2 \times 2}\right) \\
& +\frac{1}{2} \int_{\Omega} \mathcal{Q}_{2}\left(\operatorname{sym} \nabla w+\frac{1}{2} \nabla v \otimes \nabla v-\frac{1}{2}\left(\epsilon_{g}\right)_{2 \times 2}\right),
\end{aligned}
\end{aligned}
$$

with the quadratic nondegenerate form $\mathcal{Q}_{2}$, acting on matrices $F \in \mathbb{R}^{2 \times 2}$ : $\mathcal{Q}_{2}(F)=\min \left\{\mathcal{Q}_{3}(\tilde{F}) ; \tilde{F} \in \mathbb{R}^{3 \times 3}, \tilde{F}_{2 \times 2}=F\right\} ; \quad \mathcal{Q}_{3}(\tilde{F})=D^{2} W(\mathrm{Id})(\tilde{F} \otimes \tilde{F})$. 
The two terms in (8.3) measure: the first order in $h$ change of $I I$, and the second order change in $I$, under the deformation $i d+h v e_{3}+h^{2} w$ of $\Omega$. Moreover, any sequence of deformations $u^{h}$ with $I_{W}^{h}\left(u^{h}\right) \leq C h^{4}$ is, asymptotically, of this form.

9. The prestrained von Kármán equations. For the elastic energy $W$ satisfying (1.3) which is additionally isotropic (that is $W(F R)=$ $W(F)$ for all $F \in \mathbb{R}^{3 \times 3}$ and $\left.R \in S O(3)\right)$ one can see [6] that the quadratic form $\mathcal{Q}_{2}$ in the expression of $\mathcal{I}_{4}$ is given explicitly as:

$$
\mathcal{Q}_{2}\left(F_{2 \times 2}\right)=2 \mu\left|\operatorname{sym} F_{2 \times 2}\right|^{2}+\frac{2 \mu \lambda}{2 \mu+\lambda}\left|\operatorname{tr} F_{2 \times 2}\right|^{2},
$$

Here, $t r$ stands for the trace of a quadratic matrix, and $\mu$ and $\lambda$ are the Lamé constants, satisfying: $\mu \geq 0,3 \lambda+\mu \geq 0$.

Under these conditions, we rigorously derived the new von-Kármánlike system proposed in [18], written in terms of the out-of-plane displacement $v$ and the Airy stress potential $\Phi$. They describe the leading order displacements in a thin tissue which tries to adapt itself to an internally imposed metric $\left(a^{h}\right)^{T} a^{h}$ of the form (8.1):

$$
\Delta^{2} \Phi=-S\left(K_{G}+\lambda_{g}\right), \quad B \Delta^{2} v=[v, \Phi]-B \Omega_{g} .
$$

Here $S=\mu(3 \lambda+2 \mu) /(\lambda+\mu)$ is the Young's modulus, $K_{G}$ the Gaussian curvature, $B=S /\left(12\left(1-\nu^{2}\right)\right)$ the bending stiffness, and $\nu=\lambda /(2(\lambda+\mu))$ the Poisson ratio given in terms of the Lamé constants $\lambda$ and $\mu$. The corrections due to the prestrain are: $\lambda_{g}=\operatorname{curl}^{T} \operatorname{curl}\left(\epsilon_{g}\right)_{2 \times 2}$, and $\Omega_{g}=$ $\operatorname{div}^{T} \operatorname{div}\left(\left(\gamma_{g}\right)_{2 \times 2}+\nu \operatorname{cof}\left(\gamma_{g}\right)_{2 \times 2}\right)$. More precisely, the Euler-Lagrange equations of $\mathcal{I}_{4}$ are equivalent, under a change of variables which replaces the in-plane displacement $w$ by the Airy stress $\Phi$, to :

$$
\begin{aligned}
\Delta^{2} \Phi & =-S\left(\operatorname{det} \nabla^{2} v+\operatorname{curl}^{T} \operatorname{curl}\left(\epsilon_{g}\right)_{2 \times 2}\right) \\
B \Delta^{2} v & =\operatorname{cof} \nabla^{2} \Phi: \nabla^{2} v-B \operatorname{div}^{T} \operatorname{div}\left(\left(\gamma_{g}\right)_{2 \times 2}+\nu \operatorname{cof}\left(\gamma_{g}\right)_{2 \times 2}\right) .
\end{aligned}
$$

The related (free) boundary conditions on $\partial \Omega$ are:

$$
\begin{aligned}
& \Phi=\partial_{\vec{n}} \Phi=0 \\
& \tilde{\Psi}:(\vec{n} \otimes \vec{n})+\nu \tilde{\Psi}:(\tau \otimes \tau)=0 \\
& (1-\nu) \partial_{\tau}(\tilde{\Psi}:(\vec{n} \otimes \tau))+\operatorname{div}(\tilde{\Psi}+\nu \operatorname{cof} \tilde{\Psi}) \vec{n}=0
\end{aligned}
$$

where $\vec{n}$ is the normal, $\tau$ the tangent to $\partial \Omega$, and: $\tilde{\Psi}=\nabla^{2} v+\operatorname{sym}\left(\gamma_{g}\right)_{2 \times 2}$. If additionally $\partial \Omega$ is a polygonal, then the above equations simplify to the equations (5) in [18]. 
10. Derivation of the elastic shell theories. We now turn to introducing some new results and a conjecture on the variational derivation of shell theories. They can be seen as generalizations of the results in [6], justifying a hierarchy of theories for nonlinearly elastic plates. This hierarchy corresponded to scaling of the applied forces (resulting in a scaling of the energy) in terms of thickness $h$, in the limit as $h \rightarrow 0$.

We consider a compact, connected, oriented 2 d surface $S \subset \mathbb{R}^{3}$ with unit normal $\vec{n}$, and a family $\left\{S^{h}\right\}$ of thin shells around $S$ :

$$
S^{h}=\{x+t \vec{n}(x) ; \quad x \in S, \quad-h / 2<t<h / 2\}, \quad 0<h<<1 .
$$

The elastic energy of a deformation $u^{h} \in W^{1,2}\left(S^{h}, \mathbb{R}^{3}\right)$ and its total energy in presence of applied force $f^{h} \in L^{2}\left(S^{h}, \mathbb{R}^{3}\right)$ are given, respectively, by:

$$
E^{h}\left(u^{h}\right)=\frac{1}{h} \int_{S^{h}} W\left(\nabla u^{h}\right), \quad J^{h}\left(u^{h}\right)=E^{h}\left(u^{h}\right)-\frac{1}{h} \int_{S^{h}} f^{h} u^{h}
$$

where $W$ is the nonnegative stored-energy density as in (1.3).

It can be shown [6] that if $f^{h}$ scale like $h^{\alpha}$, then $E^{h}\left(u^{h}\right)$ at (approximate) minimizers $u^{h}$ of $J^{h}$ scale like $h^{\beta}$, with $\beta=\alpha$ if $0 \leq \alpha \leq 2$ and $\beta=2 \alpha-2$ if $\alpha>2$. The main part of the analysis consists now of identifying the $\Gamma$-limit $\mathcal{I}_{\beta, S}$ of $h^{-\beta} E^{h}$ as $h \rightarrow 0$, for a given scaling $\beta \geq 0$, without making any a priori assumptions on the form of the deformations $u^{h}$.

In the case when $S \subset \mathbb{R}^{2}$ (i.e. a plate), such $\Gamma$-convergence was first established for $\beta=0$ [11], and later [5, 6] for all $\beta \geq 2$. This last regime corresponds to a rigid behavior of the elastic material, since the limiting admissible deformations are isometric immersions (if $\beta=2$ ) or infinitesimal isometries (if $\beta>2$ ) of the mid-plate $S$. One particular case is $\beta=4$, where the derived limiting theory turns out to be the von Kármán theory [9]. The case $0<\beta<5 / 3$ was recently treated in [2], while the regime $5 / 3 \leq \beta<2$ remains open and is conjectured to be relevant for crumpling [21].

Much less was known when $S$ is a surface of arbitrary geometry. The first result in [11] derived the membrane theory $(\beta=0)$ where the limit $\mathcal{I}_{0, S}$ depends only on the stretching and shearing produced by the deformation. Another study [4] analyzed the case $\beta=2$, which reduces to the flexural shell model [1], i.e. a geometrically nonlinear purely bending theory, constrained to the isometric immersions of $S$. The energy $\mathcal{I}_{2, S}$ depends then on the change of curvature produced by such deformation.

11. Infinite hierarchy of shell theories. In [17] we derived (by means of asymptotic expansion) a precise conjecture on the form of $\mathcal{I}_{\beta, S}$ for $\beta>2$ and arbitrary mid-surface $S$. Namely, $\mathcal{I}_{\beta, S}$ acts on the space of $N$-th order infinitesimal isometries $\mathcal{V}_{N}$, where $N$ is such that:

$$
\beta \in\left[\beta_{N+1}, \beta_{N}\right) \quad \text { and } \quad \beta_{k}=2+2 / k .
$$

The space $\mathcal{V}_{N}$ consists of $N$-tuples $\left(V_{1}, \ldots, V_{N}\right)$ of displacements $V_{i}: S \rightarrow$ $\mathbb{R}^{3}$ (with appropriate regularity), such that the deformations $u_{\epsilon}=\mathrm{id}+$ 
$\sum_{i=1}^{N} \epsilon^{i} V_{i}$ preserve the metric on $S$ up to order $\epsilon^{N}$, i.e. $\left(\nabla u_{\epsilon}\right)^{T} \nabla u_{\epsilon}-\mathrm{Id}=$ $\mathcal{O}\left(\epsilon^{N+1}\right)$. Further, setting $\epsilon=h^{\beta / 2-1}$, we have:

(i) When $\beta=\beta_{N+1}$ then $\mathcal{I}_{\beta, S}=\int_{S} \mathcal{Q}_{2}\left(x, \delta_{N+1} I_{S}\right)+\int_{S} \mathcal{Q}_{2}\left(x, \delta_{1} I I_{S}\right)$, where $\delta_{N+1} I_{S}$ is the change of metric on $S$ of the order $\epsilon^{N+1}$, generated by the family of deformations $u_{\epsilon}$ and $\delta_{1} I I_{S}$ is the first order (i.e. order $\epsilon$ ) change in the second fundamental form.

(ii) When $\beta \in\left(\beta_{N+1}, \beta_{N}\right)$ then $\mathcal{I}_{\beta, S}=\int_{S} \mathcal{Q}_{2}\left(x, \delta_{1} I I_{S}\right)$.

(iii) The constraint of $N$-th order isometry $\mathcal{V}_{N}$ may be relaxed to that of $\mathcal{V}_{M}, M<N$, if $S$ has the following $M \mapsto N$ matching property. For every $\left(V_{1}, \ldots V_{M}\right) \in \mathcal{V}_{M}$ there exist sequences of corrections $V_{M+1}^{\epsilon}, \ldots V_{N}^{\epsilon}$, uniformly bounded in $\epsilon$, such that: $\tilde{u}_{\epsilon}=\mathrm{id}+\sum_{i=1}^{M} \epsilon^{i} V_{i}+\sum_{i=M+1}^{N} \epsilon^{i} V_{i}^{\epsilon}$ preserve the metric on $S$ up to order $\epsilon^{N}$.

This conjecture is supported by all the rigorously derived models. In particular, since plates enjoy the $2 \mapsto \infty$ matching property (i.e. [6] every $W^{1, \infty} \cap W^{2,2}$ member of $\mathcal{V}_{2}$ may be matched to an exact isometry, in the sense of (iii) above), all the plate theories for $\beta \in(2,4)$ indeed collapse to a single theory (linearized Kirchhoff model of [6]).

12. The von Kármán theory for arbitrary shells. For $\beta=4$, we proved $[13,14]$ that the $\Gamma$-limit $\mathcal{I}_{4, S}$ of $h^{-4} E^{h}$ acts on the 1-st order isometries $V \in \mathcal{V}_{1} \cap W^{2,2}$ (hence the tensor field $A=\nabla V \in W^{1,2}(S, s o(3))$ ) and finite strains $B \in \mathcal{B}=\operatorname{cl}_{L^{2}}\left\{\operatorname{sym} \nabla w ; w \in W^{1,2}\left(S, \mathbb{R}^{3}\right)\right\}$ :

$\mathcal{I}_{4, S}(V, B)=\frac{1}{2} \int_{S} \mathcal{Q}_{2}(x)\left(B-\frac{1}{2}\left(A^{2}\right)_{\text {tan }}\right)+\frac{1}{24} \int_{S} \mathcal{Q}_{2}(x)\left((\nabla(A \vec{n})-A I I)_{\text {tan }}\right)$

The two terms above correspond, in appearing order, to the stretching (2nd order change in metric) and bending (1-st order change in the second fundamental form $I I=\nabla \vec{n}$ on $S$ ) energies of a sequence of deformations $v^{h}=\mathrm{id}+h V+h^{2} w^{h}$ of $S$, which is induced by a first order displacement $V \in \mathcal{V}_{1}$ and second order displacements $w^{h}$ satisfying $\lim _{h \rightarrow 0} \operatorname{sym} \nabla w^{h}=B$.

We see that the out-of-plane displacements $v$ (present in (8.3) or other plate theories) are replaced by the vector fields in $\mathcal{V}_{1}$; neither normal, nor tangential to $S$, but instead preserving the metric up to 1-st order.

13. The case of elliptic shells. It turns out that elliptic (i.e. strictly convex up to the boundary) surfaces enjoy a stronger than plates matching property of $1 \mapsto \infty$. Namely [15], for $S$ elliptic, homeomorphic to a disk and $\mathcal{C}^{3, \alpha}(\alpha>0)$ the following holds. Given $V \in \mathcal{V}_{1} \cap \mathcal{C}^{2, \alpha}(\bar{S})$, there exists a sequence $w_{\epsilon}$, equibounded in $\mathcal{C}^{2, \alpha}\left(\bar{S}, \mathbb{R}^{3}\right)$, and such that for all small $\epsilon>0$ the field $u_{\epsilon}=\mathrm{id}+\epsilon V+\epsilon^{2} w_{\epsilon}$ is an (exact) isometry.

Regarding the assumed above regularity of $V$ (which is higher that the expected regularity $W^{2,2}$ of a limiting displacement) we note that the usual mollification techniques do not guarantee the density of smooth infinitesimal isometries in $\mathcal{V}_{1} \cap W^{2,2}$, even for $S \in \mathcal{C}^{\infty}$. This is shown by an 
interesting example of Cohn-Vossen's surface [20], which is closed smooth and of non-negative curvature, and for which $\mathcal{C}^{\infty} \cap \mathcal{V}_{1}$ consists only of trivial fields with constant gradient, whereas $\mathcal{C}^{2} \cap \mathcal{V}_{1}$ contains non-trivial elements.

However, we have the following result [15], valid for elliptic $S \in \mathcal{C}^{m+2, \alpha}$ $(\alpha \in(0,1)$ and integer $m>0)$. For every $V \in \mathcal{V}_{1} \cap W^{2,2}$ there exists a sequence $V_{n} \in \mathcal{V}_{1} \cap \mathcal{C}^{m, \alpha}\left(\bar{S}, \mathbb{R}^{3}\right)$ such that: $\lim _{n \rightarrow \infty}\left\|V_{n}-V\right\|_{W^{2,2}(S)}=0$. In the proof of the quoted results we adapted techniques of Nirenberg used for Weyl's problem (immersability of all positive curvature metrics on $S^{2}$ ).

Ultimately, and as a consequence of the above statements, the main result of [15] asserts that for elliptic surfaces with sufficient regularity, the $\Gamma$-limit of the nonlinear elastic energies $h^{-\beta} E^{h}$ for any scaling regime $\beta>2$ is given by the bending functional constrained to the 1-st order isometries:

$$
\mathcal{I}_{\text {lin }}(V)=\frac{1}{24} \int_{S} \mathcal{Q}_{2}(x)\left((\nabla(A \vec{n})-A I I)_{\text {tan }}\right), \quad A=\nabla V, \quad V \in \mathcal{V}_{1}
$$

14. Open problems. Here we list some open questions of diverse level of difficulty:

(a) identify scaling laws and $\Gamma$-limits of the $3 \mathrm{~d}$ nonlinear energy functional (1.2) for other growth tensors than these considered in [16] and [12],

(b) derive the full range hierarchy of the limiting theories, whose order will depend on the scaling of curvature tensors of a general target metric,

(c) incorporate the feedback from the minimizing configuration (shape of the thin film) back to growth mechanism (growth tensor) and study the related time-dependent (quasistatic) problem,

$(d)$ study existence, uniqueness and regularity of the critical points of the obtained models, including both minimizers and the non-minimizing equilibria (relating to the buckling phenomena),

(e) discuss convergence of non-minimizing equlibria to those of the derived models (which does not follow directly from $\Gamma$-convergence),

$(f)$ derive convergence of balance laws of the evolutionary non-Euclidean elasticity problem to the lower dimensional models,

$(g)$ justify the $3 \mathrm{~d}$ continuous growth formalism rigorously from first principles, perhaps starting from the discrete model,

(h) study the implications of result in section 4 to existence of $W^{2,2}$ regular isometric immersions for a wide class of metrics,

$(\alpha)$ validate the conjecture by deriving limiting theories for $\beta \in(2,4)$ and other than convex types of surfaces; one difficulty here is to identify the elements $V \in \mathcal{V}_{1}$ which satisfy the 2 nd order isometry condition $A^{2} \in \mathcal{B}$,

$(\beta)$ study matching, density and regularity of infinitesimal isometries (of different orders) on non-convex surfaces; e.g. hyperbolic or changing type; the analysis involves systems of PDEs of the same types,

$(\gamma)$ give an example of $S$ without flat regions and without the $1 \mapsto 2$ matching property; it will have the feature that $\mathcal{I}_{4, S}$ is again of the form as in section 12 , rather than $\mathcal{I}_{\text {lin }}$; such surfaces are expected to be non-generic. 
$(\delta)$ derive limiting theories for clamped shells; in the elliptic case this will imply that $\mathcal{V}_{k}=\{0\}$ for all $k$.

$(\epsilon)$ validate the obtained results for shells with varying thickness.

\section{REFERENCES}

[1] P.G. Ciarlet: Mathematical Elasticity, North-Holland, Amsterdam (2000).

[2] S. Conti And F. MaGGi: Confining thin sheets and folding paper, Arch. Ration. Mech. Anal. 187 (2008), no. 1, 1-48.

[3] E. Efrati, E. Sharon and R. Kupferman: Elastic theory of unconstrained nonEuclidean plates, J. Mechanics and Physics of Solids, 57 (2009), 762-775.

[4] G. Friesecke, R. James, M.G. Mora and S. Müller: Derivation of nonlinear bending theory for shells from three-dimensional nonlinear elasticity by Gamma-convergence, C. R. Math. Acad. Sci. Paris, 336 (2003), no. 8, 697702 .

[5] G. Friesecke, R. James And S. Müller: A theorem on geometric rigidity and the derivation of nonlinear plate theory from three dimensional elasticity, Comm. Pure. Appl. Math., 55 (2002), 1461-1506.

[6] G. Friesecke, R. James and S. Müller: A hierarchy of plate models derived from nonlinear elasticity by gamma-convergence, Arch. Ration. Mech. Anal., 180 (2006), no. 2, 183-236.

[7] M. Gromov: Partial Differential Relations, Springer-Verlag, Berin-Heidelberg, (1986).

[8] Q. Han And J.-X. Hong: Isometric embedding of Riemannian manifolds in Euclidean spaces, Mathematical Surveys and Monographs, 130 AMS, Providence, RI (2006).

[9] T. von KÁRmán: Festigkeitsprobleme im Maschinenbau, in Encyclopädie der Mathematischen Wissenschaften. Vol. IV/4, pp. 311-385, Leipzig, 1910.

[10] Y. Klein, E. Efrati And E. Sharon: Shaping of elastic sheets by prescription of non-Euclidean metrics, Science, 315 (2007), 1116-1120.

[11] H. Le Dret And A. Raoult: The membrane shell model in nonlinear elasticity: a variational asymptotic derivation, J. Nonlinear Sci., 6 (1996), 59-84.

[12] M. Lewicka, L. Mahadevan And M.R. PAKZAD: Morphogenesis of growing elastic tissues: the rigorous derivation of von Kármán equations with residual stress, submitted.

[13] M. Lewicka, M.G. Mora And M.R. Pakzad: Shell theories arising as low energy $\Gamma$-limit of $3 d$ nonlinear elasticity, to appear in Ann. Scuola Norm. Sup. Pisa Cl. Sci. (2009).

[14] M. Lewicka, M.G. Mora and M.R. Pakzad,: A nonlinear theory for shells with slowly varying thickness, C.R. Acad. Sci. Paris, Ser I 347 (2009), 211-216

[15] M. Lewicka, M.G. Mora AND M.R. PAKZAD: The matching property of infinitesimal isometries on elliptic surfaces and elasticity of thin shells, submitted (2008).

[16] M. Lewicka AND M.R. PAKzad: Scaling laws for non-Euclidean plates and the $W^{2,2}$ isometric immersions of Riemannian metrics, submitted (2009).

[17] M. LEWICKA AND M.R. PAKZAD: The infinite hierarchy of elastic shell models; some recent results and a conjecture, submitted (2009).

[18] L. Mahadevan and H. Liang: The shape of a long leaf, Proc. Nat. Acad. Sci. (2009).

[19] E.K. Rodriguez, A. Hoger And A. McCulloch: J.Biomechanics 27, 455 (1994).

[20] M. SpIVAK: A Comprehensive Introduction to Differential Geometry, Vol V, 2nd edition, Publish or Perish Inc. (1979).

[21] S. Venkataramani: Lower bounds for the energy in a crumpled elastic sheet - a minimal ridge, Nonlinearity 17 (2004), no. 1, 301-312. 